\title{
New anthropometric data for preoperative planning in orbital wall fracture treatment: the use of eyelid drooping
}

Han Byul Lee,

Soo Hyang Lee

Department of Plastic and

Reconstructive Surgery, Inje University

Ilsan Paik Hospital, Inje University

College of Medicine, Goyang, Korea
Background: The presence of enophthalmos is an important determinant in the decision of orbital wall fracture surgery. We proposed eyelid drooping as a new anthropometric diagnostic measure and analyzed whether eyelid drooping is associated with enophthalmos.

Methods: This retrospective study was performed from January 2014 to December 2016. A total of 75 patients with blowout fractures were studied. One experimenter measured the degree of enophthalmos using a Hertel exophthalmometer at 1 week after trauma and at 3 months after surgery. The height change of the upper eyelid was measured using the marginal reflex distance (MRD) on both sides, and the degree of eyelid drooping was calculated by comparing the two lengths. We analyzed statistically the correlation between enophthalmos and eyelid drooping.

Results: We found a highly significant correlation between the degree of enophthalmos and the reduction rate of MRD (RRM, as an indicator of eyelid drooping) at 1 week after trauma ( $r=0.845$ ). Approximately $2.0 \mathrm{~mm}$ of enophthalmos was associated with a $30.8 \%$ reduction in MRD on the affected side as compared with the normal side. At 3 months after surgery, patients showed improved eyelid appearance, with a moderate association between enophthalmos and RRM.

Conclusion: We demonstrated that the degree of enophthalmos, measured using an exophthalmometer, is associated with a change in the height of the upper eyelid. Thus, upper eyelid drooping can be used as another indicator for orbital wall fracture surgery. Compared with conventional methods, measurements of eyelid drooping are easy to perform, offering a great advantage and understanding to the patient.

Keywords: Anthropometry / Enophthalmos / Orbital fractures

\section{INTRODUCTION}

Enophthalmos, the posterior displacement of the globe along the anteroposterior axis, is a common sign of orbital wall fracture [1]. Clinically, more than $2 \mathrm{~mm}$ enophthalmos is notice-

\footnotetext{
Correspondence: Soo Hyang Lee

Department of Plastic and Reconstructive Surgery, Inje University Ilsan Paik Hospital, Inje University College of Medicine, 170 Juhwa-ro, Ilsanseo-gu, Goyang 10380, Korea

E-mail: shyanglee@naver.com

Received August 22, 2018 / Revised October 17, 2018 / Accepted October 17, 2018
}

able and a $5 \%$ increase in the total volume of the orbital cavity is sufficient to result in significant enophthalmos [2]. Other general signs of orbital wall fracture include pain, eyelid swelling, and ecchymosis, and limitations in extraocular muscle motion and radiological evidence of extensive fracture (fracture size $>2 \mathrm{~cm}^{2}$ ) are indications for surgical treatment [3]. However, the degree of enophthalmos may be masked initially by periorbital swelling, and this may result in delayed surgery or later complications [4]. Therefore, previous studies have sought to identify ways to reduce the frequency of later complications by 
measuring or predicting enophthalmos.

Hertel exophthalmometry, invented by Hertel in 1905 [5], is a simple, rapid, and easy method to measure the degree of enophthalmos. The exophthalmometer measures the distance between the lateral orbital rim and the most anterior position of the cornea. However, the method is often associated with low accuracy and poor reproducibility $[5,6]$, due to (1) improper location of the footplate on the lateral orbital rim, (2) rotation of the instrument at the horizontal plane, or (3) parallax error [7]. Nevertheless, it is widely used in clinical practice because of its simplicity, and accuracy can be improved if the device is used by an experienced examiner.

As an alternative to Hertel exophthalmometry, there have been many attempts to predict late enophthalmos by measuring orbital volume changes with preoperative computed tomography (CT). Reconstruction using three-dimensional (3D) CT is the most suitable for measuring enophthalmos because it allows for an accurate reconstruction of the complex orbital cavity $[8,9]$. However, this procedure requires specialist software to calculate the orbital volume, is costly, and time-consuming and, therefore, difficult to apply in clinical practice. In contrast, twodimensional CT is a relative inexpensive, fast, and easy to analyze method, but perhaps less accurate. There is still no consensus as to the technique of choice [10,11].

For approximately 10 years, we have managed patients with orbital wall fractures and used the aforementioned methods to predict enophthalmos. During the follow-up period, we paid particular attention to the height of the upper eyelid of the affected side, which we noted to be lower than that of the uninjured side in most patients. We surmised that this was because the eye moves posteriorly following the trauma, thereby creating a droopy lid appearance to the upper eye lid. This phenomenon is called pseudoptosis, a false ptosis because the lid function is not the problem. To date, there has been no statistical analysis on the relationship between enophthalmos and eyelid drooping. Therefore, the purpose of this study was to identify a correlation between enophthalmos and eyelid drooping in patients with orbital wall fracture. This could provide a meaningful anthropometric measure for preoperative planning in patients with orbital wall fractures.

\section{METHODS}

\section{Study design}

This retrospective cohort study was performed at the Inje University Ilsan Paik Hospital from January 2014 to December 2016. We reviewed all medical records of patients diagnosed with unilateral orbital wall fracture, and 75 patients who satis- fied the following criteria were selected: (1) "pure" orbital wall fracture (not combined with other facial bone fracture or laceration), (2) without functional impairment requiring immediate surgery, (3) without known upper eyelid height difference (inferred from patient history and previous photographs), (4) without severe peri orbital swelling that will not subside by 1 week after trauma, (5) without green stick fracture in child (requiring the surgical treatment, but usually not inducing the enophthalmos), (6) without severe hematoma (especially, with maintaining mucosal continuity in sinus).

\section{Ophthalmic examination}

All patients underwent ophthalmic examination on the first day of trauma, at 1 week after trauma (before surgery), and at 3 months after surgery. The degree of enophthalmos was measured by one skilled physician (HBL) using a Hertel exophthalmometer, according to the guidelines [5,6]: (1) Patients were positioned upright, with the patient's eye level at the examiner's eye level. (2) The precise location of the lateral orbital rim was marked using a surgical pen. (3) The device was positioned parallel to the horizontal plane. (4) The device was held tightly so it did not move with movement from the examiner. (5) The examiner should be reading the device as far from the instrument surface.

Next, the height change of the upper eyelid was analyzed using the concept of "marginal reflex distance" (MRD) (Fig. 1). MRD is the distance between the cornea light reflex and the center of the upper eyelid at the primary position. MRD was

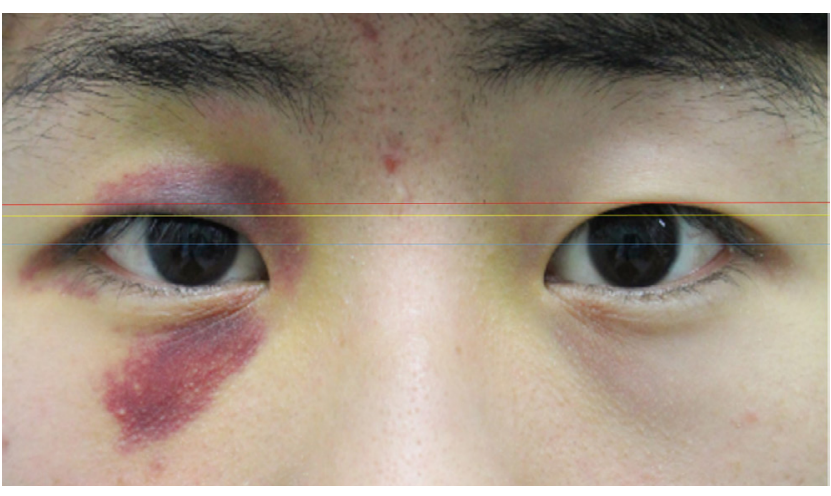

Fig. 1. Description of the measurements, using the concept of marginal reflex distance (MRD). MRD is the distance between the cornea light reflex and the center of the upper eyelid at primary position. A 25-year-old man was in a car accident and diagnosed with right orbital floor fracture. We analyzed the upper eyelid height difference between both sides at 7 days after trauma. The blue line extends between the centers of both pupils. The distance between the blue and yellow line indicates the MRD of traumatized side, whereas the distance between blue and red lines indicates the MRD of the normal side. The patient showed an MRD reduction of $15 \%$ compared with the normal side. 
measured on both sides, and the degree of eyelid drooping was calculated by comparing the two lengths. The degree of eyelid drooping is referred to as the reduction rate of the MRD (RRM).

\section{Statistical analysis}

Statistical analysis to confirm the relationship between the degree of the enophthalmos and the degree of eyelid drooping (i.e., RRM) was performed using the Pearson correlation coefficient. All analyses were carried out using IBM SPSS ver. 25.0 (IBM Corp., Armonk, NY, USA).

\section{RESULTS}

A total of 75 patients ( 45 males and 30 females) with orbital wall fractures participated in this study. The mean age of the patients was $34.9 \pm 11.3$ years (range, 14 to 67 years). The right side orbital wall was fractured in 39 cases and the left in 36 cases. An isolated orbital floor fracture was the most common (35 cases), followed by isolated medial orbital wall fracture (30 cases) and inferomedial wall combined fracture (10 cases). All patients had normal levator function after trauma (lid excursion test $\geq 10 \mathrm{~mm}$; mean \pm standard deviation $[\mathrm{SD}], 12.8 \pm 1.3 \mathrm{~mm}$; range, 10 to $15 \mathrm{~mm}$ ), and the period from injury to surgery was 7 to 19 days (mean \pm SD, $10.0 \pm 3.0$ days). The mean degree of enophthalmos at 1 week after trauma was $1.09 \mathrm{~mm}$ (SD, 0.76 $\mathrm{mm}$; range, 0 to $3 \mathrm{~mm}$ ), and the mean RRM was $13.9 \%$ (SD, $11.9 \%$; range, $0 \%$ to $55.0 \%$ ). After 3 months, the mean degree of enophthalmos was $0.35 \mathrm{~mm}$ (SD, $0.41 \mathrm{~mm}$; range, 0 to $1.5 \mathrm{~mm}$ ), and the mean RRM was $5.8 \%$ (SD, $7.5 \%$; range, $0 \%$ to $25.0 \%$ ).

There was a highly significant correlation between the degree of enophthalmos and the RRM at 1 week after trauma ( $r=$ 0.845 ) before surgical intervention, with an enophthalmos of approximately $2.0 \mathrm{~mm}$ occurring in association with a $30.8 \%$ RRM. In addition, we confirmed that the degree of enophthalmos and the RRM significantly improved with orbital wall reconstruction, noting a statistically significant effect on the recovery of enophthalmos and upper eyelid height $(p<0.05)$. Indeed, at 3 months after surgery, a moderate significant correla-

Table 1. Statistical correlation analysis on the degree of enophthalmos and RRM

\begin{tabular}{llll}
\hline Variable & Statistical method & Value & $p$-value \\
\hline$E_{1} \& R_{1}$ & Pearson correlation & $r=0.845$ & 0.001 \\
$E_{1} \& E_{p}$ & Paired $t$-test & $t=11.979$ & 0.001 \\
$R_{1} \& R_{p}$ & Paired $t$-test & $t=6.842$ & 0.01 \\
$E_{p} \& R_{p}$ & Pearson correlation & $r=0.553$ & 0.01 \\
\hline
\end{tabular}

RRM, reduction rate of marginal reflex distance; $E_{1}$, enophthalmos at 1 week after trauma; $R_{1}, R R M$ at 1 week after trauma; $E_{p}$, postoperative enophthalmos at 3 months; $R_{p}$, postoperative RRM at 3 months; $r$, correlation coefficient. tion between enophthalmos and the RRM was shown ( $r=$ 0.553) (Table 1).

Using the linear regression analysis, we could induce a functional formula between the degree of enophthalmos $(\mathrm{mm})$ and the RRM at 1 week after trauma (\%), which allows us to predict mathematically the enophthalmos using preoperative RRM measurements. Based on the following formula,

Enophthalmos $=0.0536 \times \mathrm{RRM}+0.344$

enophthalmos of approximately $2.0 \mathrm{~mm}$ would occur in association with $30.8 \%$ RRM.

Using Pearson correlation analysis, we statistically evaluated the degree of enophthalmos and the RRM at 1 week after trauma and at 3 months after surgery ( $r>0.7$ indicates high significance, $r<0.4$ indicates low significance). We found that the degree of enophthalmos and the RRM were statistically improved following orbital wall reconstruction.

Here are two cases in which this phenomenon has been appeared typically. In the first case, a 23-year-old woman was diagnosed with left orbital medial wall fracture (Fig. 2). She showed a postoperative improvement of both RRM (31.5\% to $15.5 \%)$ and the enophthalmos ( $2.0 \mathrm{~mm}$ to $0.0 \mathrm{~mm}$ ). Next, a 42 -year-old woman was diagnosed with right inferomedial orbital wall fracture (Fig. 3). She also showed an improvement of both RRM (27\% to $12 \%)$ and enophthalmos (1.5 $\mathrm{mm}$ to 0.5 $\mathrm{mm}$ ) respectively.

\section{DISCUSSION}

Orbital fractures are very common during facial trauma, and physicians should decide on a treatment plan at diagnosis. The general indications for orbital wall fracture surgery are diplopia caused by entrapment of the rectus muscle, visual acuity deficits caused by optic canal compression, and enophthalmos [3,12]. However, when patients do not show any complications, the physician must determine whether to perform surgery by evaluating preoperative $\mathrm{CT}$ images and/or through the use of enophthalmos measurements [13].

Generally, most physicians recommend performing operative reconstruction of orbital wall fractures within 2 weeks of trauma before bone union begins [14]. However, enophthalmos may be masked during the acute phase due to periorbital swelling and measured inaccurately if the examiner is not well trained. In some cases, patients do not understand the meaning of enophthalmos and refuse surgery. Therefore, many studies have been conducted to analyze the relationship between preoperative CT images and enophthalmos to predict the final outcomes for patients with enophthalmos. At present, the general consensus is that enophthalmos is caused by an increase in 

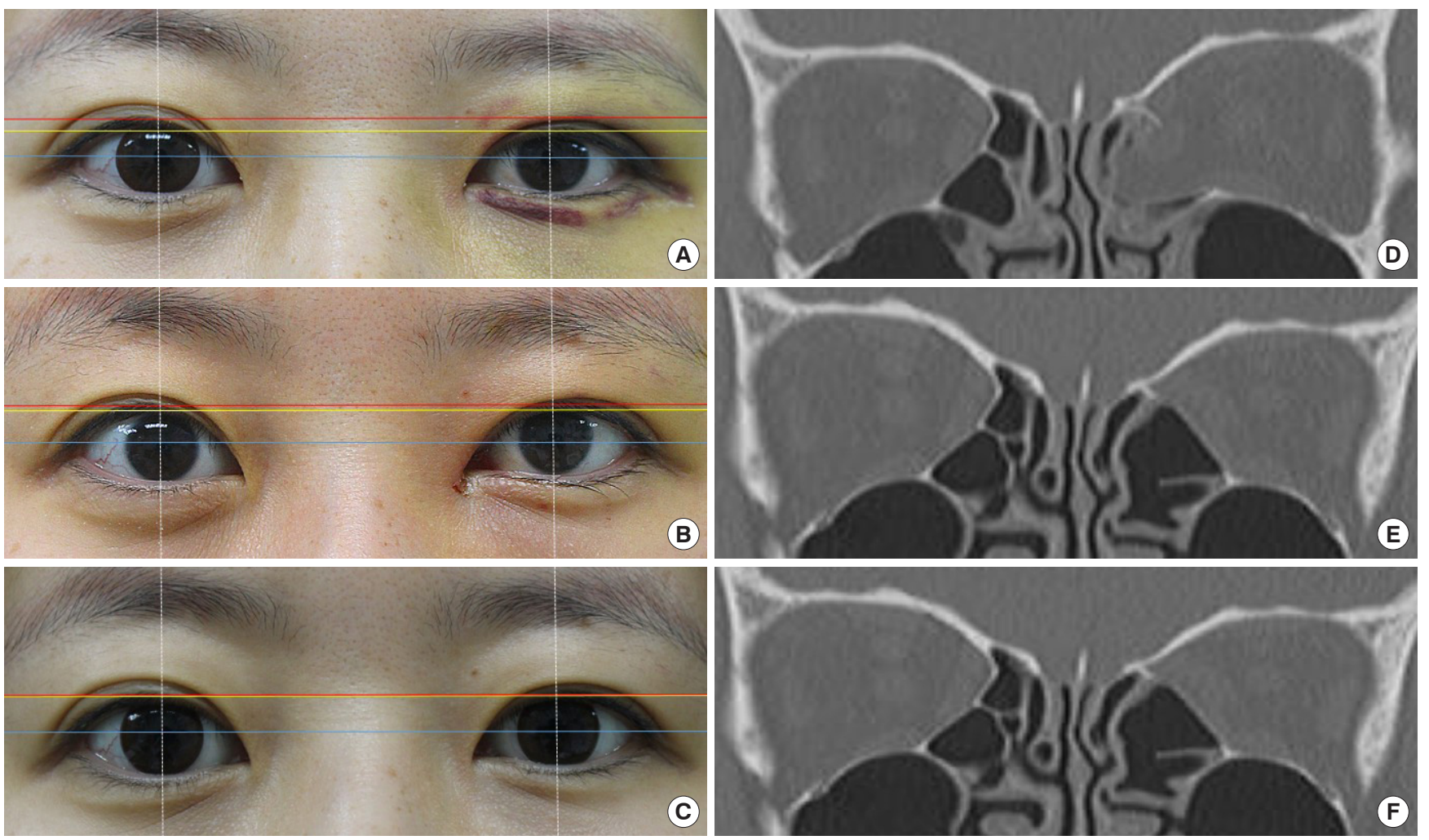

Fig. 2. Case 1, a 23-year-old woman diagnosed with left orbital medial wall fracture. Before and after surgical intervention. (A) On day 7 after trauma, reduction rate of MRD (RRM) of 31.5\% and an enophthalmos of $2.0 \mathrm{~mm}$ were shown. (B) On day 9, orbital wall reconstruction surgery was performed, which lowered the RRM to $15.5 \%$ and enophthalmos to $0.5 \mathrm{~mm}$. (C) At 3 months after surgery, eyelid height recovered to almost the same level as normal side (RRM was 3\%), and the enophthalmos was restored to zero. The blue line extends between the centers of both pupils. The distance between the blue and yellow line indicates the MRD of traumatized side, whereas the distance between blue and red lines indicates the MRD of the normal side. Two-dimensional computed tomography images taken at the time of injury (D), immediately after surgery $(\mathrm{E})$, and at 3 months after surgery $(\mathrm{F})$.
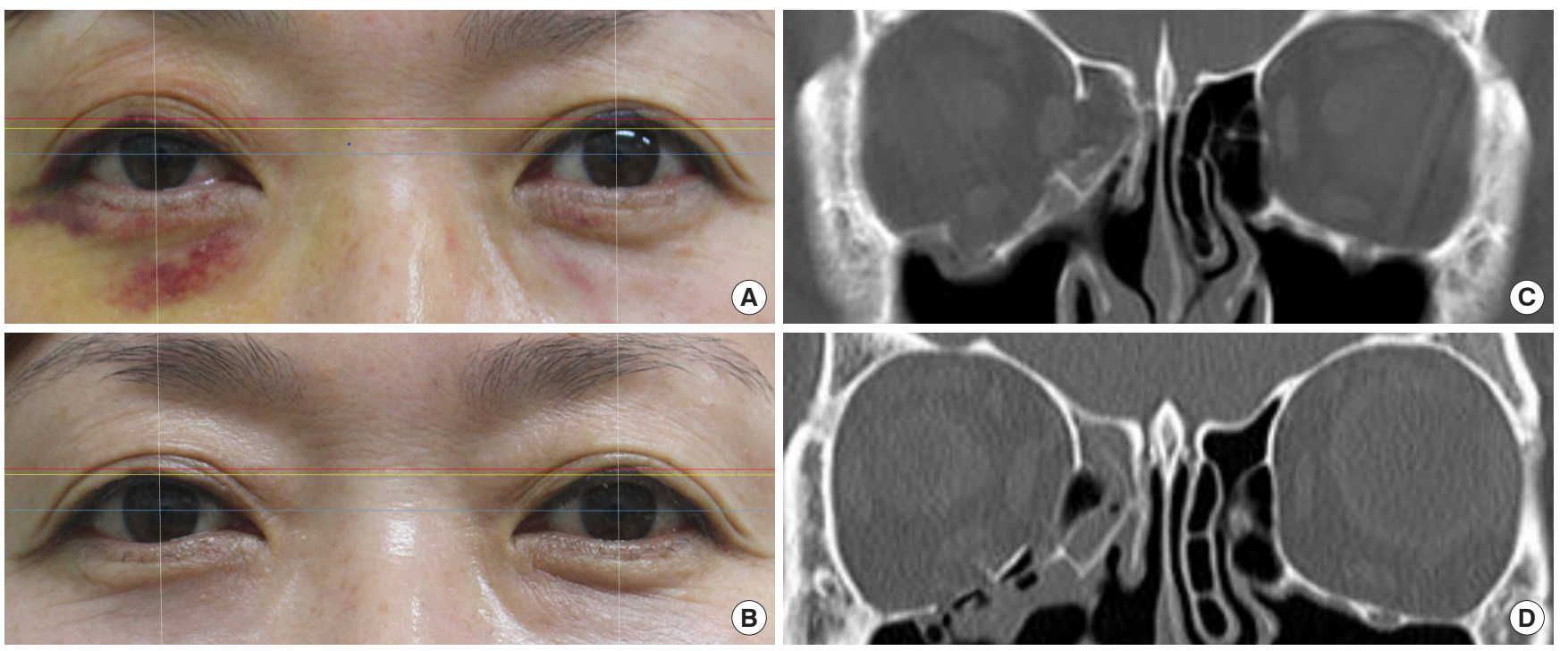

Fig. 3. Case 2, a 42-year-old woman diagnosed with right inferomedial orbital wall fracture. Before and after surgical intervention. (A) On day 7 after trauma, reduction rate of MRD (RRM) of $27 \%$ and an enophthalmos of $1.5 \mathrm{~mm}$ was shown. The patient underwent orbital wall reconstruction surgery on day 8. (B) At 3 months after surgery, RRM decreased to $12 \%$ and enophthalmos was $0.5 \mathrm{~mm}$. The distance between the blue and yellow line indicates the marginal reflex distance (MRD) of traumatized side, whereas the distance between blue and red lines indicates the MRD of the normal side. (C) Two-dimensional computed tomography image taken at the time of injury, and (D) immediately after surgery. The blue line extends between the centers of both pupils. 
orbital volume, and 3D CT reconstruction can predict enophthalmos by measuring volume change. However, because this technology is time-consuming, costly, and requires specialist equipment, its practical application is limited.

The basis of all diagnoses begins with phenomenology. Patients with orbital fractures usually have a ptotic, droopy eyelid and a smaller palpebral fissure. In clinical practice, patients do not say that their eye seems depressed (enophthalmos) but usually complain that their eye seems to be smaller or that their eyelid is sagging (pseudoptosis). From this and our previous observations, we sought to determine if the degree of eyelid drooping could be quantified and linked to the degree of the enophthalmos, and thus serve as an anthropometrical diagnostic tool that can be easily understood by patients.

We retrospectively selected patients with unilateral orbital wall fracture. All patients underwent lid excursion test at 1 week after trauma to rule out levator function impairment due to the trauma; all patients had normal levator function (above $10 \mathrm{~mm}$ ). Next, we quantified the degree of eyelid drooping by calculating the reduction rate after measuring the MRD on both sides. The degree of enophthalmos was measured at the same time by one skilled physician, and was compared statistically with the RRM. We found a highly significant correlation between enophthalmos and RRM $(r=0.845)$, with an enophthalmos of approximately $2.0 \mathrm{~mm}$ occurring in association with a 30.8\% RRM. Furthermore, we showed that orbital wall reconstruction surgery had a statistically significant effect on the recovery of enophthalmos and upper eyelid height $(p<0.05)$; albeit, with a moderate significant correlation $(r=0.553)$.

Enophthalmos occurs when the eyeball moves backward and downward along the enlarged orbital space, usually caused by an orbital wall fracture. Similarly, pseudoptosis in patients with an orbital fracture is thought to occur because of the posteroinferior movement of the eyeball, leading to downward draping of the eyelid over the anterosuperior part of the orbit.

Our findings have some important implications. First, we show that eyelid drooping can be used as a new anthropometric diagnostic measure during preoperative planning for orbital wall fracture repair. Without functional complications, the major purpose of orbital wall fracture surgery is to correct a cosmetic problem (a condition in which palpebral fissure looks smaller). Physicians usually use enophthalmos to objectively quantify such a cosmetic problem. Here, we show that eyelid drooping calculated by MRD can be used for another preoperative indicator. Second, we found that eyelid drooping improves with the recovery of enophthalmos after reconstruction of the orbital wall fracture. Although the degree of correlation was moderate, statistical associations between the two indicators are now established and can be used as an objective index to evaluate the postoperative result.

However, there are some limitations on our study. First, we conducted the study with the assumption that periorbital edema improved in the first week after trauma. Pseudoptosis may be induced or obscured by remaining periorbital edema, so our study requires premise that there is no remaining periorbital edema. Second, as in the case of using a Hertel exophthalmometer, an error also may occur in measuring the MRD itself. In addition, patients may have underlying ptosis, but do not know his or her status.

Nevertheless, the finding of greatest significance in our study is that it is much simpler, and intuitive. We acknowledge calculating orbital volume change on CT and the degree of enophthalmos measured by Hertel exophthalmometer are most powerful indicators of orbital wall fracture surgery. However, our method offers significant advantages as an ancillary method, including an ease of understanding for the patient about his or her preoperative and postoperative status. This study is expected to be useful as an ancillary indicator for managing patients with orbital wall fractures in clinical practice.

\section{CONFLICT OF INTEREST}

No potential conflict of interest relevant to this article was reported.

\section{PATIENT CONSENT}

The patients provided written informed consent for the publication and the use of their images.

\section{ORCID}

$\begin{array}{ll}\text { Han Byul Lee } & \text { https://orcid.org/0000-0002-1147-7678 } \\ \text { Soo Hyang Lee } & \text { https://orcid.org/0000-0002-1679-5624 }\end{array}$

\section{REFERENCES}

1. Gart MS, Gosain AK. Evidence-based medicine: orbital floor fractures. Plast Reconstr Surg 2014;134:1345-55.

2. Bite U, Jackson IT, Forbes GS, Gehring DG. Orbital volume measurements in enophthalmos using three-dimensional CT imaging. Plast Reconstr Surg 1985;75:502-8.

3. Rodriguez ED, Dorafshar AH, Manson PN. Facial fractures. In: Neligan PC, Rodriguez ED, Losee JE, editors. Plastic surgery. Philadelphia: Elsevier Saunders; 2013. p. 49-88.

4. Ahn HB, Ryu WY, Yoo KW, Park WC, Rho SH, Lee JH, et al. 
Prediction of enophthalmos by computer-based volume measurement of orbital fractures in a Korean population. Ophthalmic Plast Reconstr Surg 2008;24:36-9.

5. Choi KJ, Lee MJ. Comparison of exophthalmos measurements: Hertel exophthalmometer versus orbital parameters in 2-dimensional computed tomography. Can J Ophthalmol 2018;53: 384-90.

6. Genders SW, Mourits DL, Jasem M, Kloos RJ, Saeed P, Mourits MP. Parallax-free exophthalmometry: a comprehensive review of the literature on clinical exophthalmometry and the introduction of the first parallax-free exophthalmometer. Orbit 2015;34:23-9.

7. Ameri H, Fenton S. Comparison of unilateral and simultaneous bilateral measurement of the globe position, using the Hertel exophthalmometer. Ophthalmic Plast Reconstr Surg 2004;20:448-51.

8. Fan X, Li J, Zhu J, Li H, Zhang D. Computer-assisted orbital volume measurement in the surgical correction of late enophthalmos caused by blowout fractures. Ophthalmic Plast Reconstr Surg 2003;19:207-11.

9. Manson PN, Grivas A, Rosenbaum A, Vannier M, Zinreich J,
Iliff N. Studies on enophthalmos: II. the measurement of orbital injuries and their treatment by quantitative computed tomography. Plast Reconstr Surg 1986;77:203-14.

10. Rhee SH, Kim TS, Song JM, Shin SH, Lee JY. Effectiveness of computed tomography for blow-out fracture. Maxillofac Plast Reconstr Surg 2014;36:273-9.

11. Jeong SH, Shin SH, Park SH, Koo SH. Measurement analytical study of computed tomography of the orbital structure in acute blow-out fracture. J Korean Soc Plast Reconstr Surg 2007;34: 44-51.

12. Eun SC, Heo CY, Baek RM, Minn KW, Chung CH, Oh SJ. Survey and review of blowout fractures. J Korean Soc Plast Reconstr Surg 2007;34:599-604.

13. Choi SH, Kang DH, Gu JH. The correlation between the orbital volume ratio and enophthalmos in unoperated blowout fractures. Arch Plast Surg 2016;43:518-22.

14. Saiepour D, Messo E, Hedlund AJ, Nowinski DJ. Radiologic and long-term clinical outcome from treatment of isolated medial orbital wall blowout fractures. J Craniofac Surg 2012;23: 1252-5. 